\title{
Puente de Colonia-Mülheim sobre el Rin: equilibrio entre la conservación del patrimonio y las estrategias de renovación de estructuras existentes
}

\section{Rhine Bridge Cologne-Mülheim: balance between engineering heritage and replacement strategies for existing highway bridges}

\author{
Wolfgang Eilzera, Michael Müller ${ }^{\mathrm{b}}$, Thomas Lehmann ${ }^{\mathrm{c}}$, \\ Rafael Rodríguez Molina ${ }^{*}$, Bastian Kratzke ${ }^{*}$ \\ ${ }^{a}$ Dipl. Ing. Leonhardt, Andrä und Partner Beratende Ingenieure VBI AG, Stuttgart, Alemania. Consejero Delegado \\ ${ }^{b}$ Dipl. Ing. Leonhardt, Andrä und Partner Beratende Ingenieure VBI AG, Stuttgart, Alemania. Director Dpto. Internacional Puentes \\ ${ }^{c}$ Dipl. Ing. Leonhardt, Andrä und Partner Beratende Ingenieure VBI AG, Stuttgart, Alemania. Inspecciones y rehabilitación Puentes \\ ${ }^{d}$ ICCP. Leonhardt, Andrä und Partner Beratende Ingenieure VBI AG, Stuttgart, Alemania. Ing. de proyectos \\ ${ }^{e}$ M. Sc. Leonhardt, Andrä und Partner Beratende Ingenieure VBI AG, Stuttgart, Alemania. Ing. de proyectos
}

Recibido el 31 de mayo de 2019; aceptado el 14 de diciembre de 2020

\section{RESUMEN}

Cerca de un 40\% de los 40000 puentes de la red federal de carreteras de Alemania fueron construidos entre los años 60 y 70, y diseñados para las cargas de tráfico de la normativa de entonces, que además no requería análisis de fatiga. Los emblemáticos puentes sobre el Rin han sufrido especialmente el incremento de cargas y volúmenes de tráfico desde los años 80 . Nos encontramos en un momento en el que es clave establecer procedimientos sistematizados para la inspección, evaluación, recálculo y en su caso refuerzo o sustitución de las estructuras existentes. Se expone el ejemplo del sistema alemán, y en particular los aprendizajes adquiridos en la evaluación y renovación del puente de Colonia-Mülheim.

C2022 Hormigón y Acero, la revista de la Asociación Española de Ingeniería Estructural (ACHE). Publicado por Cinter Divulgación Técnica S.L. Este es un artículo de acceso abierto distribuido bajo los términos de la licencia de uso Creative Commons (CC BY-NC-ND 4.0)

PALABRAS CLAVE: Inspección, mantenimiento, evaluación, monitoreo, renovación, reparación.

\section{ABSTRACT}

Around 40\% of the 40000 bridges of the German federal highway network were built between 1960 and 1970, and were designed for the traffic loads required in the codes of the time, which did not require a fatigue check. The landmark Bridges over the river Rhine have suffered specially the increase of loads and traffic volume since the 80s. We are facing the challenge of stablishing systematic procedures for the inspection, assessment, and strengthening or replacement of existing structures. The example of the German system is presented, particularly the learnings gained with the assessment and refurbishment of Cologne-Mülheim suspension Bridge..

C2022 Hormigón y Acero, the journal of the Spanish Association of Structural Engineering (ACHE). Published by Cinter Divulgación Técnica S.L. This is an open-access article distributed under the terms of the Creative Commons (CC BY-NC-ND 4.0) License

KEYWORDS: Assessment, strengthening, surveying, rating, monitoring, inspection, refurbishment.

1.

SIGNIFICADO HISTÓRICO DE LOS PUENTES SOBRE EL RIN

Desde tiempos del Imperio Romano hasta las grandes guerras del siglo XX, "der Vater Rhein" (el padre Rin) ha supuesto en tiempos de guerra un obstáculo natural de gran importancia estratégica, así como sus cruces han sido y son en tiempos de paz un nexo vital y un símbolo de reconciliación y convivencia. En la actualidad los puentes sobre el Rin son un eslabón importante de la unión de toda Europa. Cada uno de ellos son hitos de la época histórica a la que pertenecen. Cada puente cuenta su propia historia, y también desde el punto

* Persona de contacto / Corresponding author: Correo-e / e-mail: rafael.rodriguez@lap-consult.com / bastian.kratzke@lap-consult.com (Rafael Rodríguez, Bastian Kratzke) 
de vista de la evolución del diseño y la forma juegan estos puentes un importante papel. Su conservación, rehabilitación o sustitución son grandes retos para la gestión de infraestructuras y para la ingeniería actual.

El primer puente sobre el Rin del cual se tiene constancia histórica es el efímero puente de madera de múltiples vanos cortos que Julio César mandó construir cerca de Coblenza en el año 53 a.C., construido en diez días, y que el propio César mandó destruir dieciocho días después para evitar el cruce de sus enemigos [1]. La misma motivación llevó a la completa destrucción de todos los puentes sobre el Rin durante la Segunda Guerra Mundial. La reconstrucción comenzó inmediatamente después de la guerra, en medio de una gran escasez de materiales en Alemania que imponía conseguir diseños con el máximo ahorro. Esto condujo a avances e innovaciones muy importantes en cada una de las realizaciones que se llevaron a cabo en la segunda mitad del siglo, que quedaron como legado para las siguientes generaciones. Entre ellos destacan diversas contribuciones del equipo de Fritz Leonhardt y Wolfhart Andrä, como el puente colgante de Colonia-Rodenkirchen o la reconstrucción del Colonia-Mülheim tras la guerra, la serie de puentes con cajón metálico de alma llena desarrollados en la década de los años 60, o la familia de puentes atirantados en Düsseldorf.

Ya en los años 80 el aumento del volumen de tráfico hizo necesaria la ampliación de muchos de los puentes existentes, como el de Colonia-Deutz (1979) o el de Colonia-Rodenkirchen (1990). En los últimos años nos enfrentamos al reto de encontrar el equilibrio entre la conservación de estas estructuras icónicas y la renovación de la red de infraestructuras.

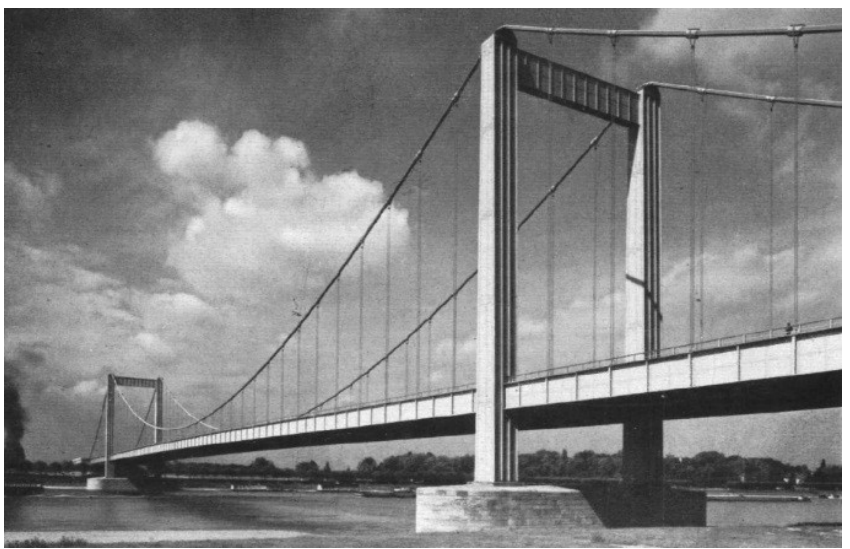

Figura 1. Puente colgante de Colonia-Rodenkirchen, antes de su destrucción en la Segunda Guerra Mundial [2]

2.

\section{SITUACIÓN ACTUAL DE LAS ESTRUCTURAS DE LA RED DE CARRETERAS EN ALEMANIA}

La mayoría de los puentes sobre el Rin más emblemáticos se integran en la red alemana de autopistas federales, que suma alrededor de $50000 \mathrm{~km}$, siendo una de las redes de mayor densidad de tráfico de Europa. El conjunto de carreteras federales de larga distancia suma casi 40000 puentes con un área total de casi 31 millones de metros cuadrados.

\subsection{Registros sobre el estado de conservación de los puentes}

La figura 2 muestra el área de puentes de la red federal de carreteras de larga distancia, según material y año, en millones de $\mathrm{m}^{2}$ (fuente: BASt [3], imagen editada para traducción y legibilidad). La Figura 3 agrupa los puentes de dicha red por año de construcción, mostrando los porcentajes del total de puentes de la red que fueron construidos en los años correspondientes a cada grupo, separando por colores los puentes de autopistas del resto de puentes de la red (fuente: BASt [3], imagen editada para traducción y legibilidad).

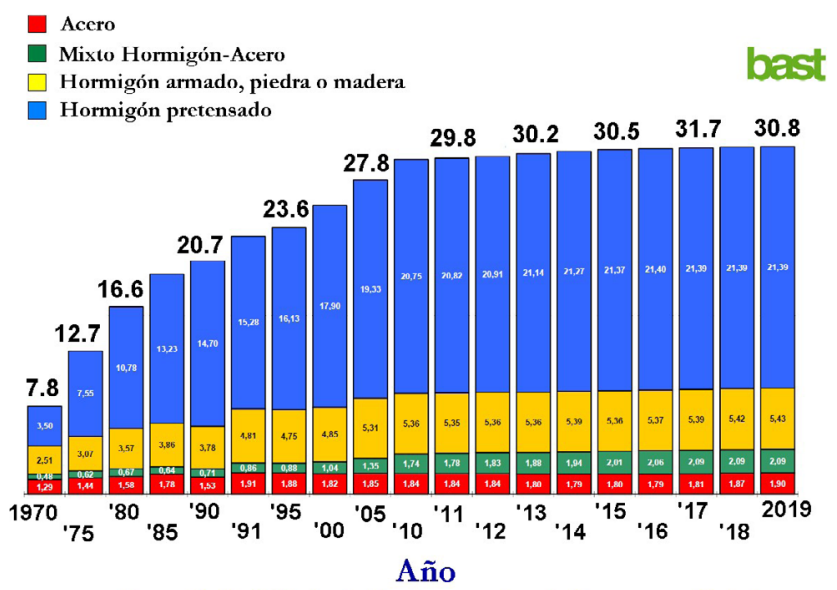

(A partir de 1991, incluidos los puentes de los nuevos Estados federales tras la Reunificación).

Figura 2. Área total de puentes de la red federal de carreteras de larga distancia.

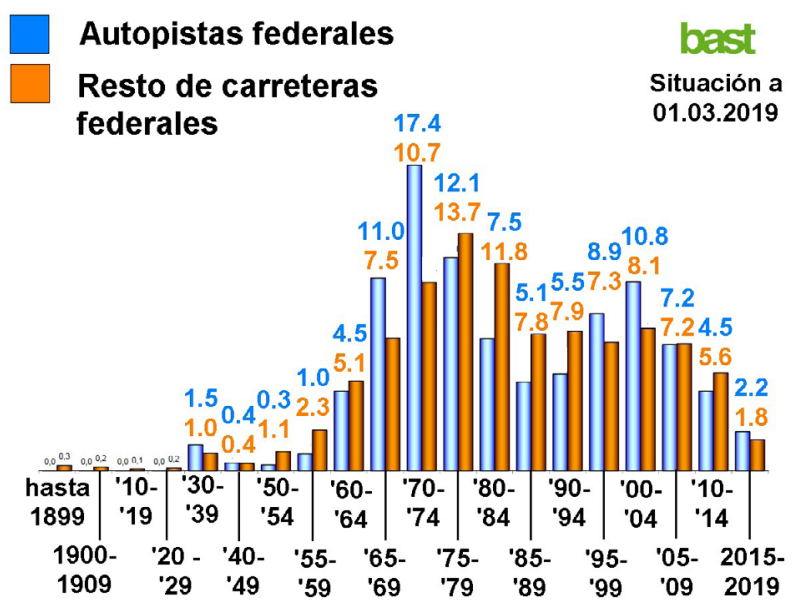

\section{Año de construcción}

Figura 3. Porcentajes del área total de puentes agrupados por año de construcción

Se puede observar que el $40 \%$ de los puentes de autopista de la red cuentan entre 50 y 60 años de antigüedad. Salvo un ligero repunte en el cambio de siglo, ambas figuras muestran que la inversión en nuevos puentes en los últimos 15 años supone solo el $14 \%$ del total de puentes existentes. Estos datos, junto con aumento tanto del volumen de tráfico como de las cargas que se han producido desde los años 80 , sugieren por sí mismos la importancia de la inversión en inspección, mantenimiento y renovación de estructuras para el futuro inmediato. La BASt (Bundesanstalt für Straßenwesen) es el ente responsable del Sistema 
de Gestión de Infraestructuras (BMS), en el cual se integra la evaluación y mantenimiento de estructuras existentes.

La norma DIN-1076 [4] establece la frecuencia y alcance de las inspecciones según los distintos elementos estructurales. Las inspecciones ordinarias se resumen en líneas generales en "Inspecciones sencillas" cada tres años e "Inspecciones principales" cada seis años, y han de ser realizadas según las "Recomendaciones para el registro, evaluación, muestra y gestión de los resultados de las inspecciones estructurales" (RI-EBW-PRÜF, [5]). Las "Inspecciones principales" son inspecciones "manuales" detalladas de todos los elementos estructurales, incluidos los de difícil acceso (interiores de cajones, pilas o pilonos en altura, apoyos) y debe incluir la apertura de elementos de cubrición para la correcta inspección de los elementos estructurales (típicamente para inspección de apoyos, anclajes de cables, etc.).

Como resultado de estas inspecciones, las estructuras reciben mediante un algoritmo estandarizado una nota (Tabla 1) asociada a su estado de seguridad estructural, seguridad del tráfico y durabilidad. En un ejercicio de llamativa transparencia, en la página web de la BASt [3] se encuentra publicada y accesible una relación de más de 50000 estructuras con sus coordenadas y la nota recibida en la última inspección.

TABLA 1.

Notas otorgadas a cada estructura tras su inspección en función de su estado de conservación.

\begin{tabular}{ll}
\hline Nota & Estado \\
\hline $1.0-1.4$ & Muy buen estado \\
$1.5-1.9$ & Buen estado \\
$2.0-2.4$ & Estado satisfactorio \\
$2.5-2.9$ & Estado suficiente \\
$3.0-3.4$ & Estado no suficiente \\
$3.5-4.0$ & Estado insuficiente \\
\hline
\end{tabular}

En la Figura 4 se aprecian los porcentajes del total de área de puentes de la red federal de carreteras que caen dentro de cada uno de los grupos por nota anteriores (Fuente: BASt, [3]. Imagen editada para traducción y legibilidad). Se puede observar que cerca del 10\% de puentes de la red han recibido una calificación de Estado no suficiente ("nicht ausreichender Bauwerkszustand") o de Estado insuficiente ("ungenügender Bauwerkszustand", el matiz de la diferencia entre ambos calificativos no es fácil de traducir).

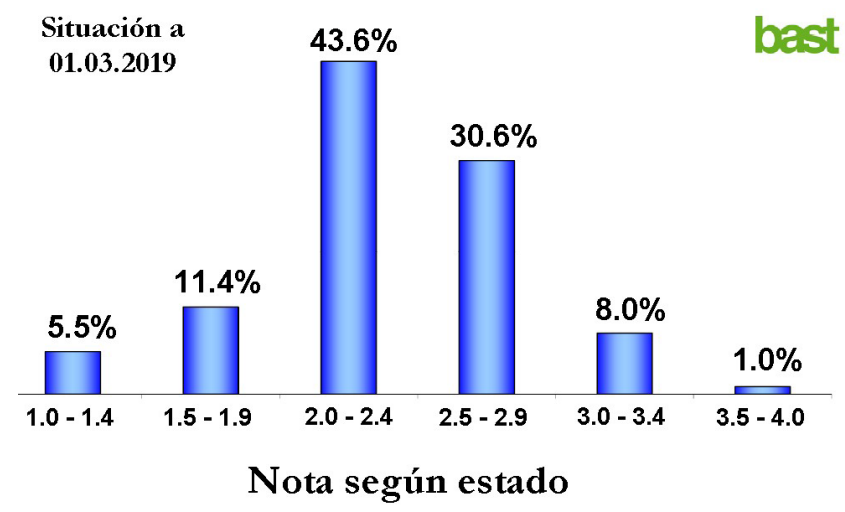

Figura 4. Porcentajes del área total de puentes de la red federal de carreteras en cada grupo de Notas según estado de conservación [3].

\subsection{Marco normativo en el campo del análisis de estructu- ras existentes}

Adicionalmente al estado externo de conservación de la estructura, es preciso un análisis de la relación entre la capacidad portante real y la demanda (normativa y/o real) en la actualidad. Ese análisis viene regulado por las Nachrechnungsrichtlinie [6] (en lo sucesivo "NaRiLi", Recomendaciones para el recálculo) que fueron publicadas en 2011 por el Ministerio federal de tráfico, construcción y desarrollo urbano (Bundesministerium für Verkehr, Bau und Stadtentwicklung), y sus posteriores adendas. Las NaRiLi marcaron un hito en esta disciplina, y proveen las directrices para la valoración realista de la capacidad portante y aptitud para el servicio de puentes de carretera existentes, incluyendo los que no fueron diseñados y construidos según las normas actuales. Adicionalmente, provee las herramientas para una toma de decisiones posterior relativamente estandarizada.

Las NaRiLi establecen un procedimiento de evaluación en cuatro niveles, que depende en primer lugar del Modelo de Cargas (Lastmodell, LM) para el cual se quiere comprobar la estructura, siendo el más exigente (el utilizado para el Nivel 1) el actual LMl de los Eurocódigos. Como se muestra en la figura 5, si la estructura satisface todas las comprobaciones de estados límite según los Eurocódigos actuales para el modelo de cargas actual, se le otorga a la estructura la Clase A. En caso de no cumplir con alguno de los requerimientos de los Estados Límite, es preciso un recálculo en segundo nivel. Las NaRiLi permiten para este Nivel 2 ciertas desviaciones de la normativa actual, como por ejemplo diferentes factores parciales de seguridad o menores ángulos de inclinación de la biela en la comprobación a cortante. Si la estructura satisface así las comprobaciones de los Estados Límite, recibe la calificación de Clase B. Si por el contrario las comprobaciones no son satisfechas, existe la posibilidad de efectuar un nuevo recálculo para un nivel de cargas reducido (como las distintas versiones de los modelos BK60 o BK30 de las versiones antiguas de las normas DIN) con la consiguiente limitación de tráfico en la estructura o reducción de su vida útil residual, recibiendo la calificación de Clase C si cumple así con los requerimientos de los Estados Límite.

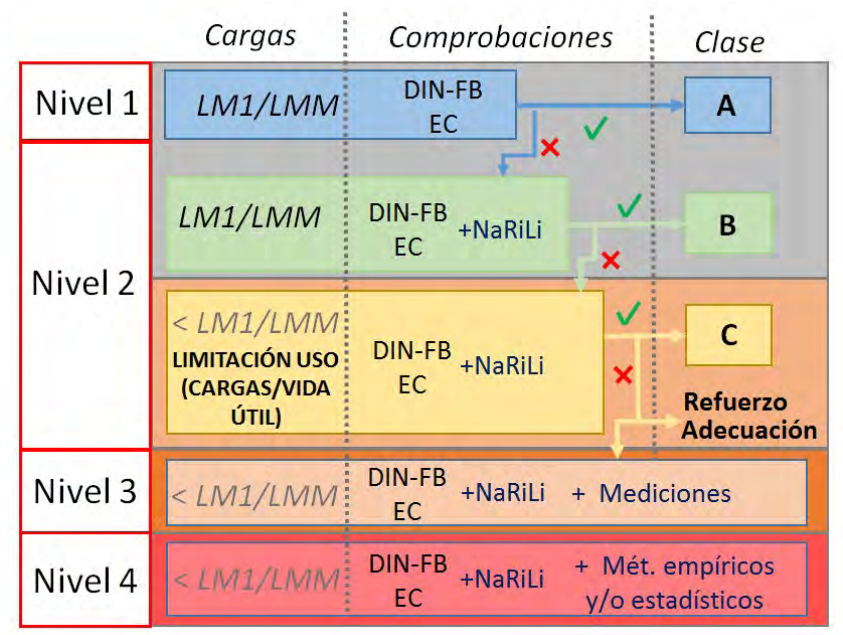

Figura 5. Distintos niveles de cálculo y Clases resultantes según las NaRiLi [6]. 
Si la estructura no supera los requerimientos para una calificación de Clase 3 es preciso un refuerzo o adecuación de la misma, tras el cual es preciso un nuevo recálculo que la sitúe en una de las tres clases anteriores, o bien se pueden llevar a cabo los niveles 3 y 4 de recálculo. Previo acuerdo de la administración y el ente propietario de la estructura, se puede efectuar el Nivel 3, para el cual se permite la consideración de los resultados de mediciones sobre la estructura (como deformaciones reales bajo las cargas actuales que solicitan la estructura, o resistencias reales deducidas de ensayos sobre probetas extraídas de la estructura). Por último, el Nivel 4 añade la posibilidad de considerar enfoques derivados de la investigación que aún no han sido integrados en la normativa.

En este marco se encuadran los casos que se exponen a continuación, que ejemplifican algunas de las experiencias de la oficina Leonhardt, Andrä und Partner (LAP) en los distintos servicios de ingeniería relacionados con el monitoreo, auscultación, mantenimiento, recálculo, refuerzo o reemplazo de puentes existentes, en este caso todos ellos sobre el Rin, que se expondrán en publicaciones paralelas a esta (figura 6, mapa tomado de [7], editado).

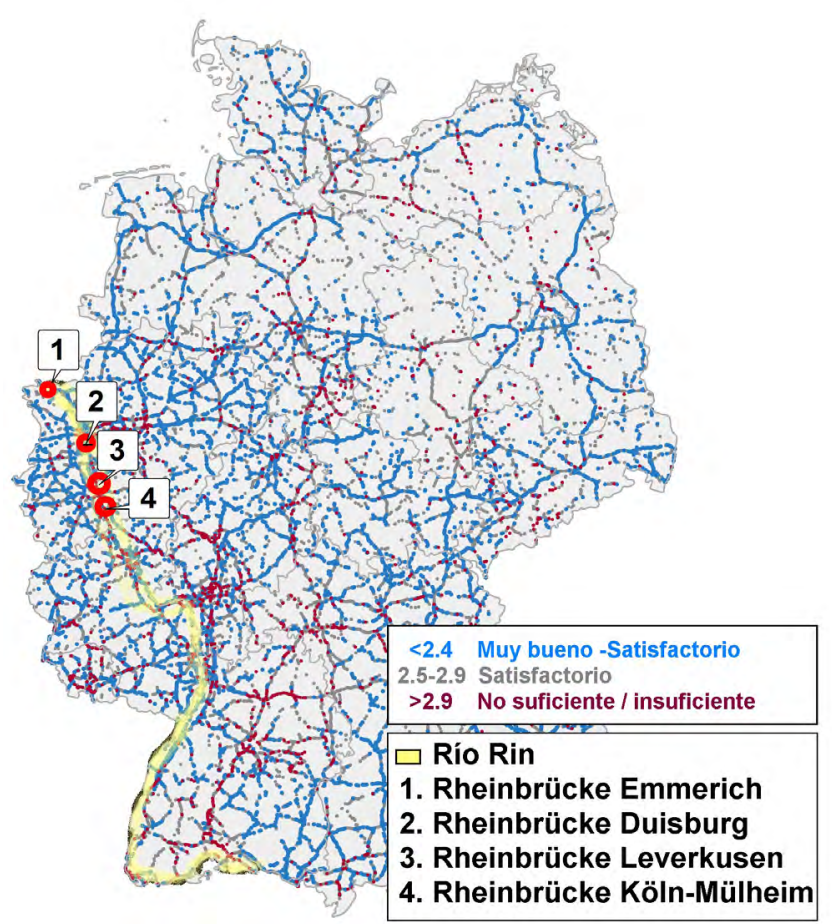

Figura 6. Puentes de la red federal según estado de conservación [7] y ubicación de los ejemplos.

3.

\section{RECÁLCULO Y ADECUACIÓN DEL PUENTE SOBRE EL RIN DE COLONIA-MÜLHEIM}

El primer puente colgante de Colonia-Mülheim fue terminado en el año 1929, pero fue destruido por un bombardeo aéreo en 1944. Su reconstrucción, según diseño de Fritz Leonhardt, se terminó en 1951 (figura 7). Se trata de un puente colgante de luces 85-315-85 metros (figura 8), con $27.74 \mathrm{~m}$ de ancho, con los cables principales anclados al terreno en los estribos. Tanto el original como el reconstruido tenían una gran similitud con el Puente de Colonia-Rodenkirchen (también proyectado por Fritz Leonhardt, este sin embargo con $378 \mathrm{~m}$ de luz), unos $10 \mathrm{~km}$ aguas arriba. El Colonia-Mülheim es el primer puente de Alemania con tablero de losa ortótropa, y llegó a ahorrar más de la mitad del acero en tablero respecto del puente original.

La sección transversal (figura 8) consta de dos vías de tranvía centrales, dos carriles de carretera a cada lado, y sendas aceras para peatones y ciclistas. Estructuralmente se resuelve con una losa ortótropa que descansa sobre vigas transversales, que transmiten las cargas a dos vigas longitudinales principales situadas en la vertical de los cables principales. En la renovación llevada a cabo en 1974 se añadieron las dos vigas de reparto longitudinales bajo los bordes de las vías de ferrocarril y los marcos de rigidización transversal.

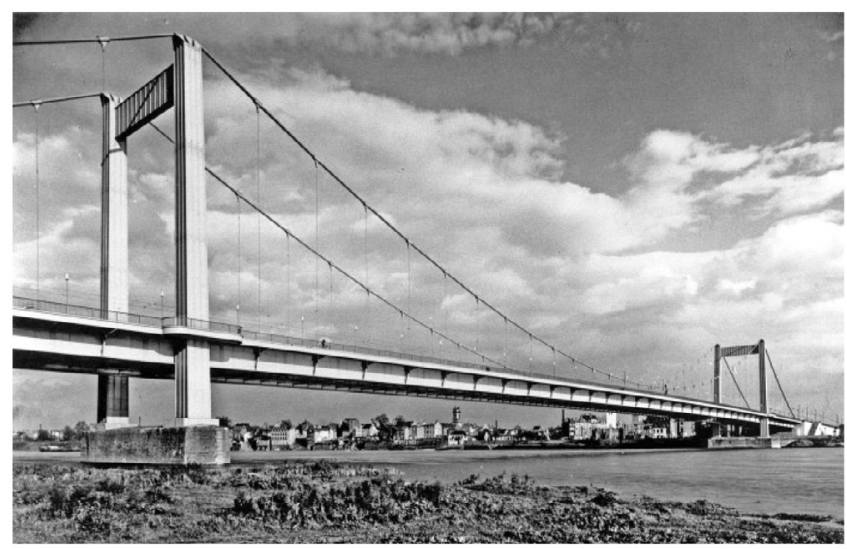

Figura 7.Vano principal del puente ColoniaMülheim en torno a 1952 (LAP)

Las inspecciones realizadas en el marco de la DIN 1076 identificaron repetidos daños en los pernos de las uniones de las vigas longitudinales de reparto añadidas en 1974 con las vigas transversales (figura 10). Dichas vigas fueron diseñadas sin tener en cuenta los esfuerzos que recibirían por colaboración en el comportamiento global del tablero. Los trabajos de recálculo en el marco de las NaRiLi y las actuaciones posteriores fueron encargadas a LAP (información más extensa en $[8,9])$.

\subsection{Recálculo de fatiga}

En un primer recálculo de Nivel 1 se identificó que a pesar de las vigas de reparto añadidas en 1974 la capacidad de las vigas transversales con anclajes de péndolas era insuficiente frente al modelo de cargas LMl. Se identificaron tensiones por encima de lo admisible en cerca de un $80 \%$ de la longitud del puente, y se identificaron incumplimientos de los estados límite de fatiga en el ala inferior de las vigas de reparto bajo el ferrocarril para el modelo de cargas de fatiga 3.

Para el recálculo de fatiga de Nivel 2 se consideró en primer lugar el Modelo de carga de fatiga 4 con acumulación de daño, para categoría de tráfico 2 (media distancia) de las NaRili [6], junto con las cargas del vagón de tranvía asumiendo una ocupación del 66\%. Sin embargo, la probabilidad de coincidencia del tráfico de carretera con el tranvía quedaba fuera 

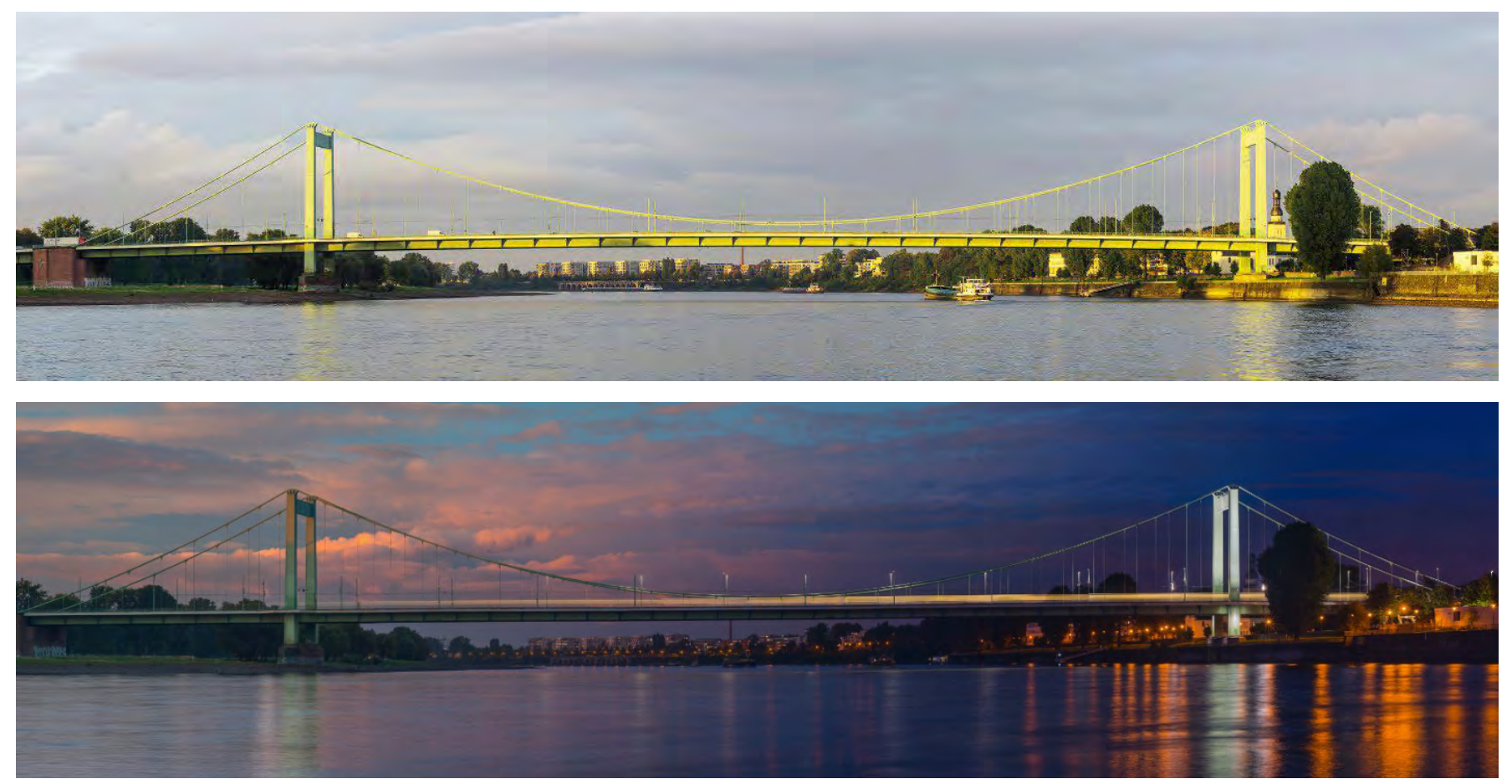

Figura 8. Puente colgante de Colonia-Mülheim, 2017 (fotografías: Bastian Kratzke).
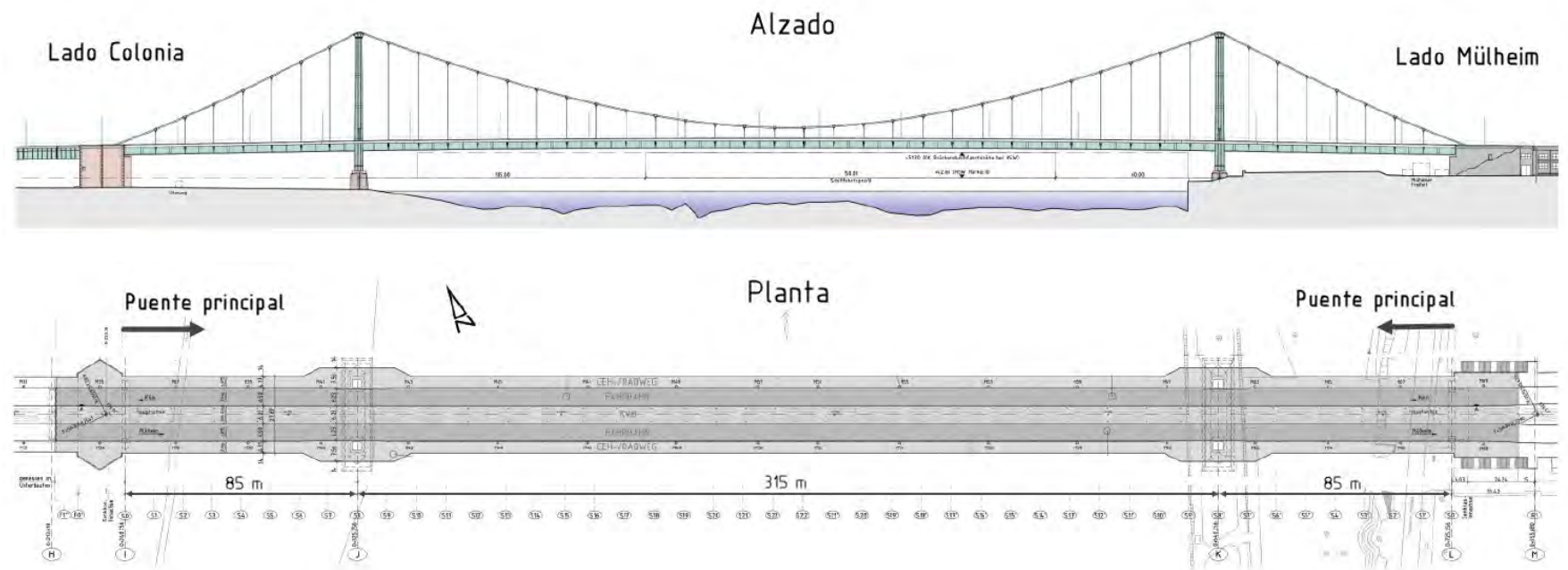

Figura 9. Alzado y planta del puente principal Colonia-Mülheim (LAP).

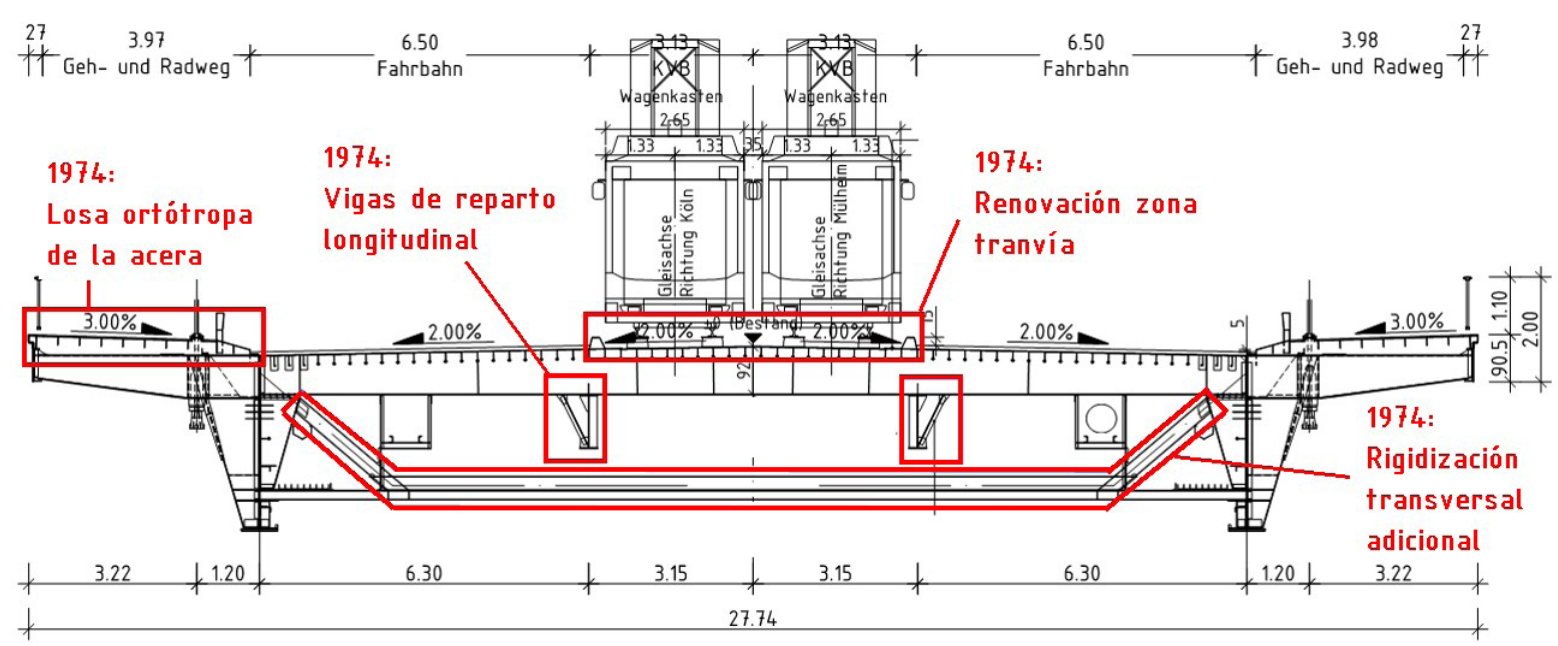

Figura 10. Sección transversal del puente Colonia-Mülheim, con los añadidos de 1974 (LAP). 

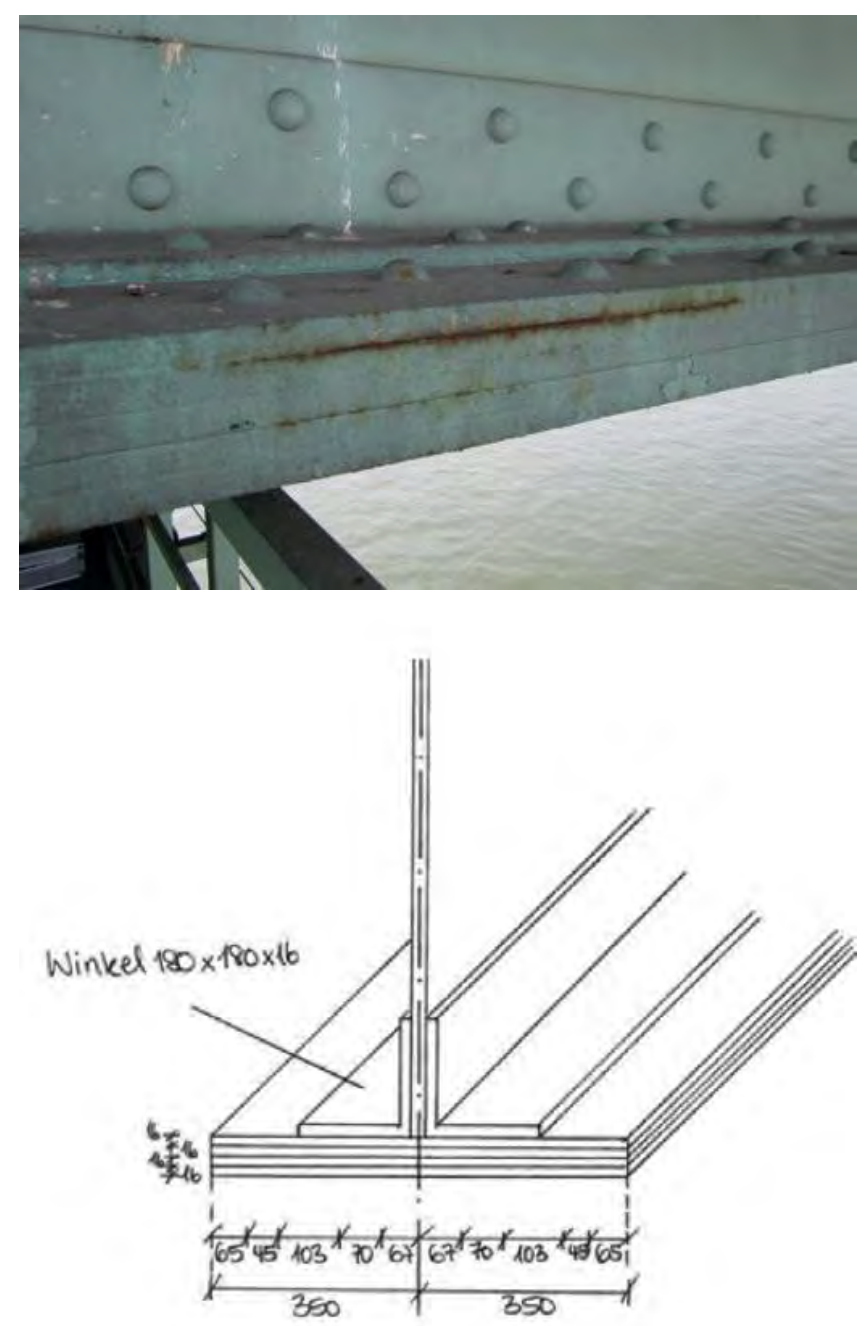

Figura 11. Ala inferior de las vigas longitudinales principales (LAP). de los escenarios contemplados por las NaRiLi. Analizando los horarios de trenes desde 1959 hasta 2013 se pudo estimar una frecuencia de 65500 trenes por vía al año (131000 para las 2 vías). Igualmente, los registros de tráfico de carretera permitían estimar alrededor de 600000 vehículos pesados del tipo 2 cada año. Ante la falta de datos combinados, se decidió asumir del lado de la seguridad la coincidencia del camión en cada paso del ferrocarril, y el resto de camiones actuando aisladamente. Se identificaron así problemas de fatiga en los marcos transversales, en las vigas transversales y las vigas longitudinales principales, especialmente en su ala inferior, que disponía perfiles angulares roblonados al alma y ala inferior (figura 11).

Tras los resultados insatisfactorios obtenidos del análisis de Nivel 2, las NaRiLi plantean, como alternativa al reemplazo de los elementos afectados, el análisis de Nivel 3 (figura 5). El nivel de daño acumulado (según el modelo de acumulación de daño de Palmgren/Miner recogido en el Eurocódigo EC1993-1-9) arrojado por los cálculos no se correspondía con las deficiencias, más limitadas, observadas en las inspecciones, por lo que se acordó con la administración propietaria del puente abordar un cálculo de Nivel 3.

Se repartieron a lo largo de distintos puntos del tablero un total de 48 extensómetros (figura 13) en los elementos que se habían mostrado deficitarios en los cálculos, y se realizaron diversas mediciones con el puente cerrado al tráfico, bajo cargas de camiones de distintos tipos, así como de trenes sobre la línea de ferrocarril, individualmente y también junto con los camiones. Adicionalmente se colocaron cámaras en el pilono, que una vez abierto el puente de nuevo al tráfico permitían identificar a tiempo real la correspondencia entre las tensiones deducidas de la medición de los extensómetros con el paso de los distintos tipos de vehículos (figura 14).

Además del análisis de los tipos de vehículos y sus frecuencias, estas mediciones permitían calibrar en el modelo las rigideces de

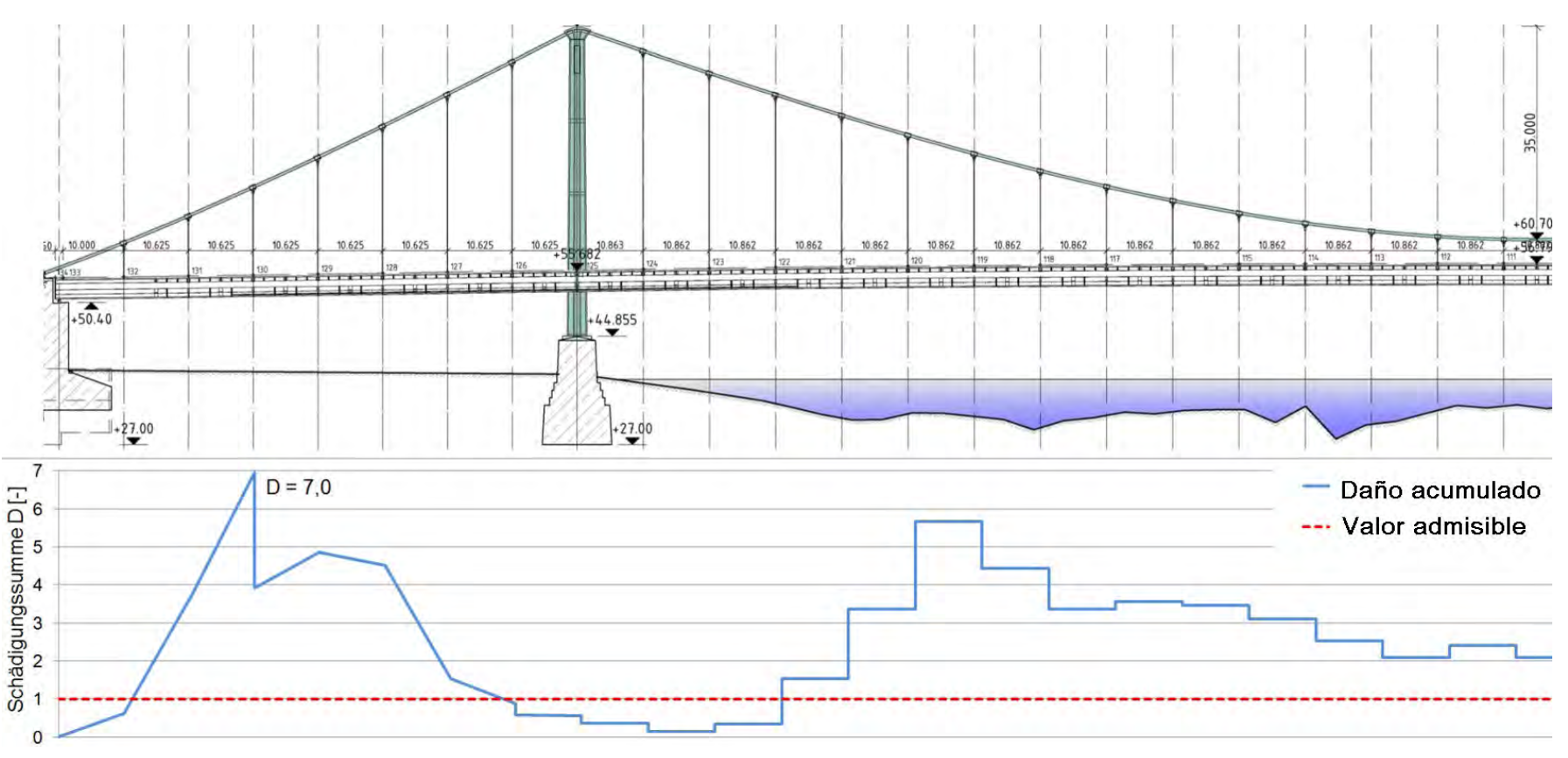

Figura 12. Daño pasado acumulado para el ala inferior de las vigas principales (LAP). 
algunas uniones y analizar las tensiones dinámicas en relación con los valores de cálculo estático. Tras el análisis de cuatro meses de mediciones se pudo llegar a la conclusión de que el modelo de cargas de fatiga considerado en el Nivel 2 era muy conservador, por lo que se decidió elaborar un modelo de carga de fatiga adaptado a la estructura a partir de datos reales registrados, lo cual plantea el reto de conseguir un modelo que cubra la realidad del lado de la seguridad, sin ser excesivamente conservador.

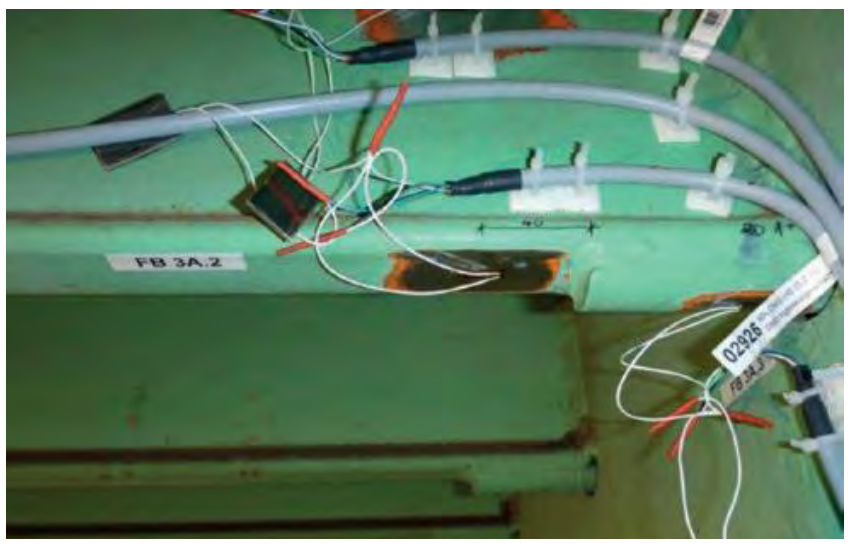

Figura 13. Extensómetros colocados en el tablero (LAP).

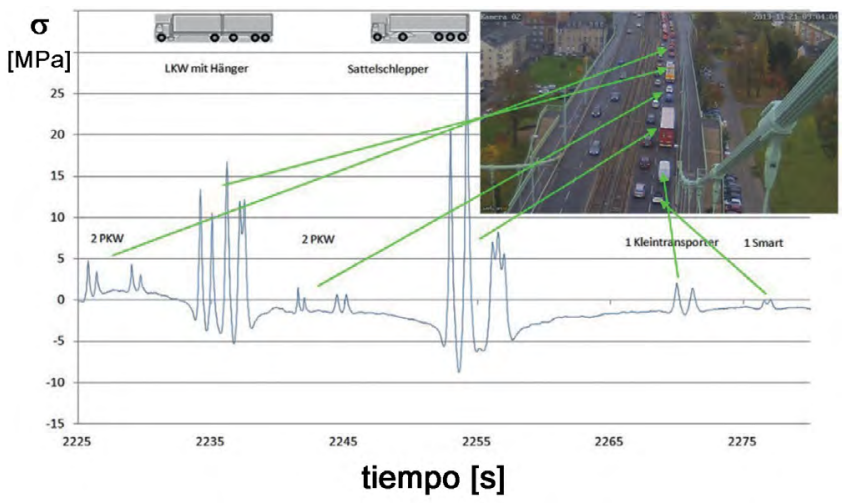

Figura 14. Análisis de tensiones registradas y correspondencia con vehículos reales (LAP).

A su vez, el modelo había de ser lo suficientemente sencillo como para hacer abordable el cálculo. Para ello se tomaron como base los mismos modelos de carga utilizados en el cálculo de Nivel 2, calibrando el número de pasadas para obtener un daño acumulado equivalente a partir de las medidas de tensiones reales registradas. Los cerca de 450 vehículos diarios registrados con pesos de entre 2.5 y 50 toneladas fueron representados por un camión de 30 toneladas con una frecuencia de 52 vehículos por día (que con el factor de seguridad de 1.5 del modelo de carga resulta en 80 camiones diarios). Para la previsión a futuro, se consideraron los tranvías un 50\% más largos como provisión ante el eventual aumento del volumen de viajeros, y se multiplicó por dos la probabilidad de ocurrencia de casos extremos como la ocupación simultánea de las dos vías.

Con este modelo de cargas de fatiga se obtuvo, para el mismo detalle crítico al que se refieren las figuras 11 y 12 , un valor de daño de $\mathrm{D}=0.24$ (comparado con el $\mathrm{D}=7.0$ del cálculo Nivel 2). Para poder aceptar semejante diferencia fue preciso hacer una evaluación realista del daño acumulado en el pasado. El análisis de los registros históricos de volumen de tráfico sobre el puente (figura 15) deja ver la influencia sobre el volumen de tráfico de distintos factores que a priori pueden escaparse del análisis. La línea roja discontinua muestra la estimación del tráfico real en el puente a lo largo de los años. Entre el año 55 y el 60 se observa una importante crecida del volumen de tráfico, interrumpida tras la apertura en 1966 del puente Zoo, menos de $3 \mathrm{~km}$ aguas arriba. La renovación de la Wiener Platz en 1992 afectó nuevamente a la distribución del tráfico y al volumen de vehículos sobre el puente. Estos volúmenes quedan muy por debajo de los establecidos por las NaRiLi para las carreteras de larga distancia (línea verde, que sí se correlaciona bien con los puntos cercanos de las autopistas BABl y BAB-3) e incluso de los valores de la NaRiLi para tráfico de proximidad (línea azul).

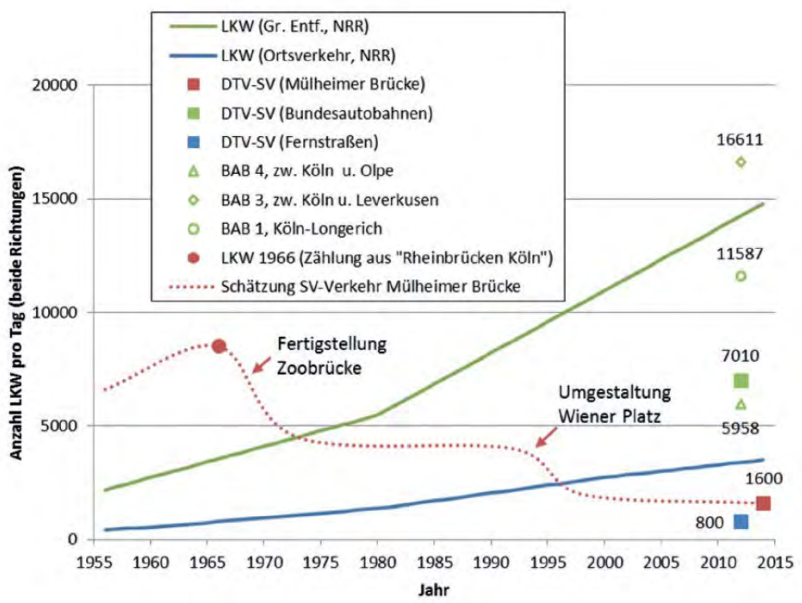

Figura 15. Volumen de tráfico sobre el puente ColoniaMülheim comparado con los establecidos en las NaRiLi [10].

\subsection{Refuerzos a ejecutar}

Además del análisis a fatiga expuesto, el resto de análisis sobre el puente (más información en [8] y [9], en alemán) llevaron a la decisión de realizar distintos refuerzos. Se decidió sustituir las vigas longitudinales de reparto añadidas en 1974 por celosías diseñadas esta vez no sólo como viga de reparto si no como elemento colaborante en el comportamiento global. Estas celosías pueden observarse ya modelizadas en la figura 16 (en un complejo modelo con la losa ortótropa modelizada mediante láminas, por la dificultad para determinar los anchos eficaces de la misma para cada caso de carga, al trabajar como ala superior de estas celosías y resto de elementos longitudinales).

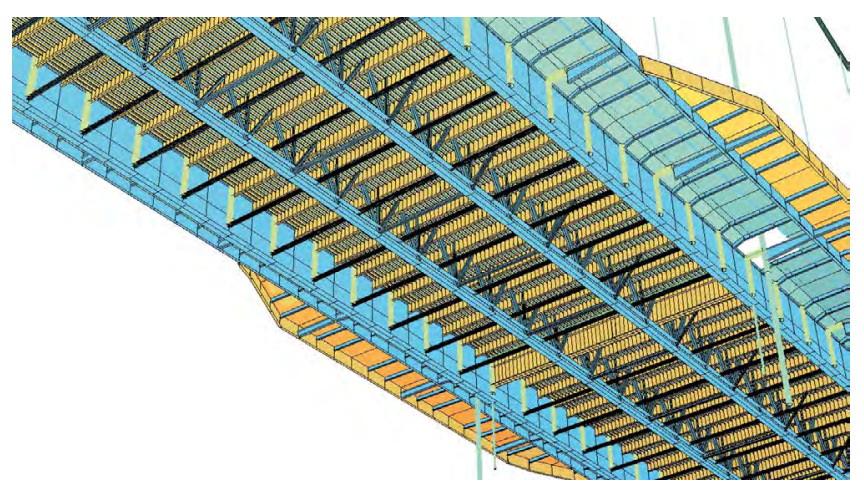

Figura 16. Modelo de cálculo integrando los elementos de refuerzo (LAP) 
Así mismo, las vigas transversales con anclaje a las péndolas necesitan también un refuerzo. Sin embargo, se optó por disminuir su solicitación a base de reforzar las vigas transversales adyacentes, para evitar que un refuerzo de las primeras resulte en una aún mayor solicitación debido al aumento de rigidez.

Se llevará a cabo una completa sustitución de todas las péndolas (de cable cerrado), manteniendo el tráfico en la estructura, sustituyendo también los anclajes al tablero por nuevos anclajes con un diseño que mejora su durabilidad y facilidad de inspección.

Por último, se renovará y ampliará la zona de las aceras en el entorno de los pilonos, y se renovarán las instalaciones de los pilonos (escaleras, plataformas, iluminación) así como la pintura de protección frente a la corrosión en todo el puente y las capas de rodadura. Se realizarán reparaciones menores, como reparaciones de fisuras detectadas en algunas soldaduras, sustitución de algunos tornillos, etc.

A falta de inspeccionar los anclajes en estribos de los cables principales (que quedaba fuera del alcance de los trabajos expuestos) las medidas propuestas conferirán al puente Colonia-Mülheim 100 años más de vida útil.

4.

\section{CONCLUSIONES}

La situación de los puentes que forman parte de las redes de infraestructuras de toda Europa hace necesaria la implementación de procedimientos sistematizados para su inspección, registro, toma de decisiones y eventual renovación o sustitución. El ejemplo del Puente Colonia-Mülheim, en el marco normativo y administrativo alemán, ilustra como un sistema de este tipo, junto con una necesaria competencia técnica y voluntad de acuerdo y trabajo en equipo de las administraciones y las oficinas de diseño, permite conservar estructuras que son hitos de la historia y la ingeniería.

\section{Referencias}

[1] L. Fernández Troyano, Tierra sobre el agua. Visión histórica universal de los puentes, CICCP, España, 1999, 171-173.

[2] F. Leonhardt et al., Die Autobahnbrücke über den Rhein bei Köln-Rodenkirchen, Ernst \& Sohn, 1952.

[3] www.BASt.de $>$ Brücken- und Ingenieurbau $>$ Statistik, Bundesanstalt für Straßenwesen - BASt, Bundesministerium für Verkehr, Bau und Stadtentwicklung, 2019.

[4] Normenausschuß Bauwesen (NaBau), DIN 1076: 1999-11, DIN Deutsches Institut für Normung e.V., 1999.

[5] Richtlinie zur einheitlichen Erfassung, Bewertung, Aufzeichnung und Auswertung von Ergebnissen der Bauwerksprüfungen nach DIN 1076 (RIEBW-PRÜF), Bundesministerium für Verkehr und digitale Infrastruktur, Alemania, 2007.

[6] Richtlinie zur Nachrechnung von Straßenbrücken im Bestand (Nachrechnungsrichtlinie), Bundesministerium für Verkehr, Bau und Stadtentwicklung, Abteilung Straßenbau, Alemania, 2011.

[7] www.welt.de/politik/interaktiv/bruecken/ deutschlands-bruecken-wettlauf-gegen-den-verfall.html, elaborado a partir de los datos de [3] para el año 2016.

[8] B. Kratzke, H. Uphoff, S. Hanke, Instandsetzung und Ertüchtigung des Mülheimer Brückenzuges in Köln, Stahlbau 87, Heft 8 (2018) 759-768.

[9] B. Kratzke, Ertüchtigung der Brücke Köln-Mülheim, 17. Symposium Brückenbau Leipzig, Ausgabe 1/2 (2017), 26-31.

[10]K. Geißler, R. Stein, A. Reichardt, Monitoring Rheinbrücke Köln-Mülheim, Messbericht der GMG Ingenieurgesellschaft mbH,. Berlin, 2014. 